\title{
Shift Operators for the Quantum Calogero-Sutherland Problems via Knizhnik-Zamolodchikov Equation
}

\author{
Giovanni Felder ${ }^{1}$, Alexander P. Veselov ${ }^{2}$ \\ ${ }^{1}$ ETH-Zentrum, CH-8092 Zürich, Switzerland \\ 2 Moscow State University, Moscow, Russia
}

Received: 17 March 1993

\begin{abstract}
We give a natural interpretation of the shift operators for CalogeroSutherland quantum problem via KZ equation using Matsuo-Cherednik mappings. The explicit formulas for the inversions of these mappings and versions of shift operators for KZ equations are also found. As an application we show that the shift operator can be described via a factorization problem for an appropriate quantum integral (discriminant) of the Calogero system.
\end{abstract}

\section{Introduction}

The Calogero system [1] describes the motion of $N$ particles on the line interacting with the potential $U_{g}^{c}(x)=g u^{c}(x)$,

$$
u^{c}(x)=\sum_{i \neq j}^{N} \frac{1}{\left(x_{i}-x_{j}\right)^{2}},
$$

$x_{i}$ are the coordinates of the points, $g$ is a coupling constant. The value of $g$ is not essential in the classical case but it is in the quantum case. For $N=2$ the corresponding Schrödinger equation

$$
\left(-\Delta+U_{g}^{c}(x)\right) \psi=E \psi,
$$

$\Delta=\partial_{1}^{2}+\cdots+\partial_{N}^{2}, \partial_{i} \equiv \partial / \partial x_{i}$, can be reduced to the one-dimensional one:

$$
\left(-\frac{d^{2}}{d y^{2}}+\frac{g}{y^{2}}\right) \varphi=\lambda \varphi, \quad y=x_{1}-x_{2} .
$$

If we introduce a new constant $k$, such that

$$
k(k-1)=g,
$$


then the operator $L_{k}=-\frac{d^{2}}{d y^{2}}+\frac{k(k-1)}{y^{2}}$ can be factorized as

$$
L_{k}=-\left(\frac{d}{d y}+\frac{k}{y}\right)\left(\frac{d}{d y}-\frac{k}{y}\right) .
$$

Let us remark that

$$
L_{k+1}=-\left(\frac{d}{d y}-\frac{k}{y}\right)\left(\frac{d}{d y}+\frac{k}{y}\right) \text {, }
$$

which implies the following relation for the operator $\mathscr{D}_{k}=\frac{d}{d y}-\frac{k}{y}$ :

$$
L_{k+1} \mathscr{D}_{k}=\mathscr{D}_{k} L_{k} .
$$

This means that the operator $\mathscr{D}_{k}$ maps (formal) eigenfunctions of the operator $L_{k}$ to eigenfunctions of $L_{k+1}$ : if $L_{k} \varphi=\lambda \varphi$ and $\tilde{\varphi}=\mathscr{D}_{k} \varphi$, then $L_{k+1} \tilde{\varphi}=L_{k+1} \mathscr{D}_{k} \varphi$ $=\mathscr{D}_{k} L_{k} \varphi=\lambda \tilde{\varphi}$.

It turns out that such operators exist for all $N$. In the most general case this was proven by Opdam [6], who considered together with Heckman the trigonometric version of Calogero system (Sutherland system) and its generalization first proposed by Olshanetsky and Perelomov (see [2-6]).

The Sutherland system describes the motion of particles on the line (circle, if $\omega$ is purely imaginary) interacting with the potential $U_{g, \omega}^{s}=g u_{\omega}^{s}$,

$$
u_{\omega}^{s}(x)=\sum_{i \neq j} \frac{\omega^{2}}{\sinh ^{2}\left(x_{i}-x_{j}\right) \omega} .
$$

In the limit $\omega \rightarrow 0$ one has the Calogero potential (0.1), so $u_{0}^{s}=u^{c}$.

Following Opdam, we will call such operators $\mathscr{D}_{k}$ shift operators. They are differential operators with highest term $\prod_{i<j}\left(\partial_{i}-\partial_{j}\right)$, which satisfy the relation

$$
L_{k+1} \mathscr{D}_{k}=\mathscr{D}_{k} L_{k}
$$

for $L_{k}=-\Delta+k(k-1) u_{\omega}^{s}, L_{k+1}=-\Delta+k(k+1) u_{\omega}^{s}$.

The explicit form of the shift operator $\mathscr{D}_{1}$ for any $N$ was first found in 1988 by Chalykh and one of the authors in [8], where the problem of supercomplete commutative rings of partial differential operators was discussed. It turned out that the shift operators $\mathscr{D}_{k}$ for integer $k$ play a very important role in this problem (see $[8,9])$. Other very interesting applications of shift operators (e.g. the proof of some of Macdonald's conjectures) were found by Opdam in [7].

In 1990 Heckman used the so-called Dunkl operator to give the explicit form of the shift operators in the most general case $[10,11]$. The Dunkl operator [12] looks very much like the differential operator of the Knizhnik-Zamolodchikov (KZ) equation, which appeared in conformal field theory in $1984[13]^{1}$. This hints at a possible relation between $\mathrm{KZ}$ and Calogero-Sutherland (CS) equations. The explicit form of such relations was discovered by Matsuo [15]. The result of Matsuo was generalized and extended by Cherednik in [19].

\footnotetext{
${ }^{1}$ We would like to remark also the resemblance of all these operators with Moser's $L$-operator for Calogero-Sutherland system (see [14])
} 
The main goal of our paper is to give a natural interpretation of shift operators for the Calogero-Sutherland quantum problem via $\mathrm{KZ}$ equation using the Matsuo-Cherednik isomorphisms. We prove the commutativity of the following diagram

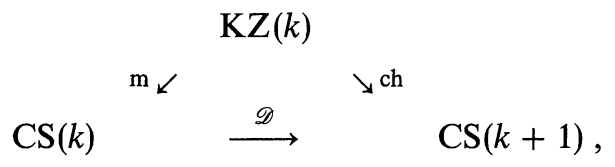

where $\mathrm{m}$ and ch denote the Matsuo and Cherednik mappings from the space of solution of $\mathrm{KZ}$ equation with parameter $k$ into that of the Calogero-Sutherland problems with parameters $k$ and $k+1$ respectively, and $\mathscr{D}$ is proportional to the shift operator $\mathscr{D}_{k}$. Generically, $\mathrm{m}$ and ch are the isomorphisms, so the shift operator is completely determined by this property. We also give a formula for the inverse $\mathrm{m}^{-1}$ of Matsuo's map, which leads to a shift operator for the KZ equation.

As an application, we show that the shift operator can be described via a factorization problem for an appropriate quantum integral (discriminant) of the Calogero system. This is related to the duality connecting solutions of the $\mathrm{KZ}$ equations with parameters $k$ and $-k$. It follows from our results that the operator $\mathscr{D}$ is conjugated to this duality by Matsuo's map.

\section{Calogero Quantum System and KZ Equation}

Let us consider the equation

$$
\partial_{i} \Phi=\left(k \sum_{j \neq i} \frac{P^{(i j)}}{z_{i}-z_{j}}+\lambda^{(i)}\right) \Phi,
$$

where $\Phi=\Phi\left(z_{1}, \ldots, z_{N}\right)$ takes values in the tensor product $V \otimes V \otimes \cdots \otimes V$ $=V^{\otimes N}$ of some vector space $V, P^{(i j)}$ is the permutation of the $i^{\text {th }}$ and $j^{\text {th }}$ factors, $\lambda$ is a diagonal matrix considered as a parameter, $\lambda^{(i)}$ is the operator in $V^{\otimes N}$ acting as $\lambda$ on $i^{\text {th }}$ factor and identically on all other factors. When $\lambda=0$ this equation coincides with that for correlation functions for WZNW model in CFT, derived by Knizhnik and Zamolodchikov in [13]. We will call (1.1) KZ equation.

Let us consider the case when $\operatorname{dim} V=N$ and $\Phi$ has the form

$$
\Phi=\sum_{\sigma \in S_{N}} \Phi_{\sigma} e_{\sigma}, \quad e_{\sigma}=e_{\sigma(1)} \otimes e_{\sigma(2)} \otimes \cdots \otimes e_{\sigma(N)},
$$

$S_{N}$ is the symmetric group. Following Matsuo [15] define the scalar function $\varphi=m(\Phi)$ as

$$
m(\Phi)=\sum_{\sigma \in S_{N}} \Phi_{\sigma}
$$

Proposition 1. If $\Phi=\sum_{\sigma \in S_{N}} \Phi_{\sigma} e_{\sigma}$ is a solution of the $\mathrm{KZ}$ equation (1.1), then $\varphi=m(\Phi)$ is eigenfunction of the Calogero operator $L_{k}^{c}$ :

$$
L_{k}^{c} \varphi:=\left(-\Delta+k(k-1) \sum_{i \neq j} \frac{1}{\left(z_{i}-z_{j}\right)^{2}}\right) \varphi=E \varphi,
$$

where $E=-\left(\lambda_{1}^{2}+\cdots+\lambda_{N}^{2}\right), \lambda=\operatorname{diag}\left(\lambda_{1}, \ldots, \lambda_{N}\right)$. 
Proof. For the components $\Phi_{\sigma}$ one derives from (1.1) the following system:

$$
\partial_{i} \Phi_{\sigma}=k \sum_{j \neq i} \frac{\Phi_{\sigma \circ p_{i j}}}{z_{i}-z_{j}}+\lambda_{\sigma(i)} \Phi_{\sigma},
$$

$p_{i j} \in S_{N}$ is the transposition of $i$ and $j$.

After summation over $\sigma \in S_{N}$ one has

$$
\partial_{i} \varphi=k \varphi \sum_{j \neq \imath} \frac{1}{\left(z_{i}-z_{j}\right)}+\chi_{i},
$$

where $\chi_{i}=\sum \lambda_{\sigma(i)} \Phi_{\sigma}$, and therefore

$$
\partial_{i}^{2} \varphi=k \sum_{j \neq i} \frac{\partial_{i} \varphi}{z_{i}-z_{j}}-k \sum_{j \neq i} \frac{\varphi}{\left(z_{i}-z_{j}\right)^{2}}+\partial_{i} \chi_{i} .
$$

In its turn

$$
\begin{aligned}
\partial_{i} \chi_{i} & =\sum_{\sigma} \lambda_{\sigma(i)} \partial_{i} \Phi_{\sigma}=k \sum_{j \neq i} \sum_{\sigma} \frac{\lambda_{\sigma(i)} \Phi_{\sigma \circ p_{i j}}}{z_{i}-z_{j}}+\sum_{\sigma} \lambda_{\sigma(i)}^{2} \Phi_{\sigma} \\
& =k \sum_{j \neq i} \frac{\sum_{\mu \in S_{N}} \lambda_{\mu(j)} \Phi_{\mu}}{z_{i}-z_{j}}+\sum_{\sigma} \lambda_{\sigma(i)}^{2} \Phi_{\sigma}=k \sum_{j \neq i} \frac{\chi_{j}}{z_{i}-z_{j}}+\sum_{\sigma} \lambda_{\sigma(i)}^{2} \Phi_{\sigma} .
\end{aligned}
$$

Finally we have

$$
\begin{aligned}
\Delta \varphi & =\sum_{i=1}^{N}\left(\partial_{i}^{2} \varphi\right)=k \sum_{j \neq i} \frac{\partial_{i} \varphi}{z_{i}-z_{j}}-k \sum_{j \neq i} \frac{\varphi}{\left(z_{i}-z_{j}\right)^{2}}+k \sum_{j \neq i} \frac{\chi_{j}}{z_{i}-z_{j}}+\sum_{i, \sigma} \lambda_{\sigma(i)}^{2} \Phi_{\sigma} \\
& =k^{2} \varphi \sum_{\substack{i, j, l \\
l \neq i \\
j \neq i}}\left(z_{i}-z_{j}\right)^{-1}\left(z_{i}-z_{l}\right)^{-1}-k \varphi \sum_{i \neq j}\left(z_{i}-z_{j}\right)^{-2}+k \sum_{i \neq j} \frac{\chi_{i}+\chi_{j}}{\left(z_{i}-z_{j}\right)}+\lambda^{2} \varphi \\
& =\left(k^{2}-k\right) \varphi \sum_{i \neq j}\left(z_{i}-z_{j}\right)^{-2}+\lambda^{2} \varphi .
\end{aligned}
$$

In the last step we have used the identities

$$
\sum_{i \neq j} \frac{\chi_{i}+\chi_{j}}{z_{i}-z_{j}} \equiv 0
$$

and

$$
\sum_{i \neq j \neq l}\left(z_{i}-z_{j}\right)^{-1}\left(z_{i}-z_{l}\right)^{-1} \equiv 0 .
$$

Proposition 1 is proven.

It turns out that there exists another relation between the $\mathrm{KZ}$ equation and the Calogero system. The following remark belongs to Cherednik [19].

Let $\psi=\operatorname{ch}(\Phi)$ for $\Phi=\sum_{\sigma} \Phi_{\sigma} e_{\sigma}$ be defined as

$$
\operatorname{ch}(\Phi)=\sum_{\sigma} \varepsilon(\sigma) \Phi_{\sigma},
$$

where $\varepsilon(\sigma)=\operatorname{sign}(\sigma)$ is the sign of permutation $\sigma$. 
Proposition 2. For any solution of the $\mathrm{KZ}$ equation (1.1) of the form (1.2) $\Phi=\sum_{\sigma} \Phi_{\sigma} e_{\sigma}$ the function $\psi=\operatorname{ch}(\Phi)$ is eigenfunction of the Calogero operator $L_{k+1}$ :

$$
\left(-\Delta+k(k+1) \sum_{i \neq j} \frac{1}{\left(z_{i}-z_{j}\right)^{2}}\right) \psi=E \psi, \quad E=-\lambda^{2} .
$$

Proof. By direct calculation:

$$
\partial_{i} \psi=\sum_{\sigma} \varepsilon(\sigma)\left(k \sum_{j \neq i} \frac{\Phi_{\sigma \circ p_{i j}}}{z_{i}-z_{j}}+\lambda_{\sigma(i)} \Phi_{\sigma}\right)=-k \psi \sum_{j \neq i} \frac{1}{\left(z_{i}-z_{j}\right)}+\sum_{\sigma} \varepsilon(\sigma) \lambda_{\sigma(l)} \Phi_{\sigma} .
$$

Introducing $\omega_{i}=\sum_{\sigma} \varepsilon(\sigma) \lambda_{\sigma(i)} \Phi_{\sigma}$ one has

$$
\partial_{i} \omega_{i}=\sum_{\sigma} \varepsilon(\sigma) \lambda_{\sigma(i)}\left(k \sum_{j \neq i} \frac{\Phi_{\sigma \circ p_{i j}}}{z_{i}-z_{j}}+\lambda_{\sigma(l)} \Phi_{\sigma}\right)=-k \sum_{j \neq i} \frac{\omega_{j}}{z_{i}-z_{j}}+\sum_{\sigma} \varepsilon(\sigma) \lambda_{\sigma(i)}^{2} \Phi_{\sigma}
$$

and

$$
\begin{aligned}
\Delta \psi= & \sum_{i=1}^{N} \partial_{i}^{2} \psi=k \psi \sum_{i \neq j} \frac{1}{\left(z_{i}-z_{j}\right)^{2}}-k \sum_{j \neq i} \frac{\partial_{i} \psi}{\left(z_{i}-z_{j}\right)}+\sum_{i} \partial_{i} \omega_{i} \\
= & k \psi \sum_{i \neq j} \frac{1}{\left(z_{i}-z_{j}\right)^{2}}-\sum_{\substack{i \neq \ell \\
l \neq \ell}} \frac{k}{z_{i}-z_{j}}\left(-\frac{k \psi}{z_{i}-z_{\ell}}+\omega_{i}\right) \\
& +\sum_{i} \sum_{j \neq i}\left(-\frac{k \omega_{j}}{z_{i}-z_{j}}\right)+\lambda^{2} \psi=\left(k^{2}+k\right) \psi \sum_{i \neq j} \frac{1}{\left(z_{i}-z_{j}\right)^{2}}+\lambda^{2} \psi .
\end{aligned}
$$

In fact from $[15,19]$ it follows that $\varphi$ and $\psi$ are eigenfunctions of the commutative ring $\mathscr{R}_{k}^{c}$ of quantum integrals of the Calogero problem,

$$
\begin{array}{ll}
\mathscr{A} \varphi & =\gamma(\mathscr{A}) \varphi, \quad \mathscr{A} \in \mathscr{R}_{k}^{c}, \\
\mathscr{A} \psi & =\gamma(\mathscr{A}) \psi, \quad \mathscr{A} \in \mathscr{R}_{k+1}^{c} .
\end{array}
$$

Here $\mathscr{R}_{k}^{c}=\left\{\mathscr{A}:\left[\mathscr{A}, L_{k}^{c}\right]=0\right.$ and $\sigma^{*}(\mathscr{A})=\mathscr{A}$ for all $\left.\sigma \in S_{N}\right\}, \gamma$ is the so-called Harish-Chandra isomorphism

$$
\gamma: \mathscr{R}_{k}^{c} \leftrightarrow \mathbb{C}[\lambda]^{S_{N}}
$$

between $\mathscr{R}_{k}^{c}$ and the ring of all symmetric polynomials $\mathbb{C}[\lambda]^{S_{N}}$ (see e.g. [10]). ${ }^{2}$ Moreover, Matsuo and Cherednik proved that for generic $\lambda$, more precisely, if all $\lambda_{i}$ are different, the mappings $\mathrm{m}$ and $\mathrm{ch}$ are isomorphisms between the space $\mathrm{KZ}(k)$ of solutions of $\mathrm{KZ}$ equation (1.1) of the form (1.2) and the spaces $C(k), C(k+1)$ of solutions of the systems (1.9), (1.10).

This means that for generic $\lambda$ we have the mapping $\mathscr{D}=\mathrm{ch} \circ \mathrm{m}^{-1}: \varphi \rightarrow \psi$ from $C(k)$ into $C(k+1)$ (see the diagram)

\section{Diagram 1}

$\mathrm{KZ}(k)$

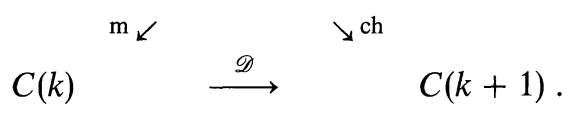

\footnotetext{
${ }^{2}$ It is interesting to note that the ring of all (not necessary $S_{N}$-invariant) quantum integrals of the Calogero problem for integer $k$ is much bigger than $\mathscr{R}_{k}^{C}$ (see $[8,9]$ )
} 
It turns out that $\mathscr{D}$ is related in a very simple way to the shift operator $\mathscr{D}_{k}$ for the Calogero system (see Introduction). Recall that $\mathscr{D}_{k}$ is the differential operator with highest order term $\delta(\partial)$,

$$
\delta(\xi)=\prod_{i<j}\left(\xi_{i}-\xi_{j}\right)
$$

and the property

$$
L_{k+1}^{c} \mathscr{D}_{k}=\mathscr{D}_{k} L_{k}^{c}
$$

Theorem 1. Let $\mathscr{D}$ be the operator

$$
\mathscr{D}=\delta^{-1}(\lambda) \mathscr{D}_{k},
$$

where $\mathscr{D}_{k}$ is the shift operator for Calogero quantum problem, then the diagram 1 is commutative.

Proof. We will use Heckman's results $[10,11]$ about the shift operator for the Calogero system via Dunkl operator.

Let $\nabla_{\xi}$ be the following operator, acting on a scalar function $\varphi=\varphi\left(z_{1}, \ldots, z_{N}\right)$,

$$
\nabla_{\xi}=\partial_{\xi}-k \sum_{j \neq i} \frac{\xi_{i}-\xi_{j}}{z_{i}-z_{j}} \hat{p}_{i j}
$$

where $\partial_{\xi}$ is the derivative in the direction $\xi$ and

$$
\left(\hat{p}_{i j} \varphi\right)(z)=\varphi\left(p_{i j}(z)\right)
$$

$p_{i j}$ as above is the transposition of the indices $i$ and $j$. The operator $\nabla_{\xi}$ is conjugated to the Dunkl operator [12] by the gauge transformation

$$
\varphi \rightarrow \varphi \prod_{i<j}\left(z_{i}-z_{j}\right)^{k}
$$

In contrast with the papers by Heckman, Opdam, Dunkl and Matsuo [5-7, 10-12] we prefer the gauge of many-body problems (cf. [4]).

G. Heckman found the following expression for the shift operator $\mathscr{D}_{k}$ :

$$
\mathscr{D}_{k}=C_{N}^{-1} \delta^{-1}(\xi) \operatorname{Res}\left(\sum_{\sigma \in S_{N}} \varepsilon(\sigma) \nabla_{\sigma(\xi)}^{d}\right), \quad d=\frac{N(N-1)}{2} .
$$

Here $C_{N}$ is some constant (see below), Res $\mathscr{A}$ means the differential part of the operator $\mathscr{A}$, which can be found by taking the restriction of $\mathscr{A}$ on the space of symmetric functions (see $[10,11]$ ). The vector $\xi$ should be taken regular, i.e.

$$
\delta(\xi)=\prod_{i<j}\left(\xi_{i}-\xi_{j}\right) \neq 0 .
$$

Thus, for given solution $\Phi=\Sigma_{\sigma} \Phi_{\sigma} e_{\sigma}$ of the $\mathrm{KZ}$ equation (1.1), we have to calculate the expression

$$
\operatorname{Res}\left(\sum_{\sigma} \varepsilon(\sigma) \nabla_{\sigma(\xi)}^{d}\right)(\varphi), \quad \varphi=\mathrm{m}(\Phi)=\Sigma \Phi_{\sigma}
$$

The following lemma can be extracted from [15].

Let $\tilde{\nabla}_{\eta}^{m}$ be $\operatorname{Res}\left(\nabla_{\eta}^{m}\right)$. Then we have

$$
\tilde{\nabla}_{\eta}^{m+1}=\operatorname{Res}\left[\left(\partial_{\eta}-k \sum_{j \neq i} \frac{\eta_{i}-\eta_{j}}{z_{i}-z_{j}} \hat{p}_{i j}\right) \circ \nabla_{\eta}^{m}\right]=\partial_{\eta} \tilde{\nabla}_{\eta}^{m}-k \sum_{j \neq i} \frac{\eta_{i}-\eta_{j}}{z_{i}-z_{j}} \tilde{\nabla}_{p_{i j}(\eta)}^{m} .
$$


Lemma. If $\Phi=\Sigma \Phi_{\sigma} e_{\sigma}$ is a solution of $\mathrm{KZ}$ equation (1.1) and $\varphi=\mathrm{m}(\Phi)=\Sigma_{\sigma} \Phi_{\sigma}$, then

$$
\tilde{\nabla}_{\eta}^{m}(\varphi)=\sum_{\sigma}(\sigma(\eta), \lambda)^{m} \Phi_{\sigma}
$$

Proof. By induction. For $m=0$ it is obvious. Suppose that it is true for all $m \leqq n$. Then

$$
\begin{aligned}
\tilde{\nabla}_{\eta}^{n+1}(\varphi)= & \left(\partial_{\eta} \tilde{\nabla}_{\eta}^{n}-k \sum_{j \neq i} \frac{\eta_{i}-\eta_{j}}{z_{i}-z_{j}} \tilde{\nabla}_{p_{i j}(\eta)}^{n}\right)(\varphi) \\
= & \partial_{\eta}\left(\sum_{\sigma}(\sigma(\eta), \lambda)^{n} \Phi_{\sigma}\right)-k \sum_{j \neq i} \frac{\eta_{i}-\eta_{j}}{z_{i}-z_{j}} \sum_{s} g\left(\left(\sigma \circ p_{i j}(\eta), \lambda\right)^{n} \Phi_{\sigma}\right) \\
\mu=\sigma \circ p_{i j} & \sum_{\sigma}(\sigma(\eta), \lambda)^{n}\left(k \sum_{i \neq j} \frac{\eta_{i}-\eta_{j}}{z_{i}-z_{j}} \Phi_{\sigma \circ p_{t j}}+(\sigma(\eta), \lambda) \Phi_{\sigma}\right) \\
& \quad-k \sum_{i \neq j} \frac{\eta_{i}-\eta_{j}}{z_{i}-z_{j}} \sum_{\mu \in S_{N}}(\mu(\eta), \lambda)^{n} \Phi_{\mu \circ p_{i j}}=\sum_{\sigma}(\sigma(\eta), \lambda)^{n+1} \Phi_{\sigma} .
\end{aligned}
$$

The lemma is proven.

Now we have

$$
\begin{aligned}
\sum_{\sigma \in S_{N}} \varepsilon(\sigma) \tilde{\nabla}_{\sigma(\xi)}^{d}(\varphi) & =\sum_{\sigma \in S_{N}} \varepsilon(\sigma)\left(\sum_{\mu \in S_{N}}(\mu(\sigma(\xi)), \lambda)^{d} \Phi_{\mu}\right) \\
\mu \sigma & =v \\
& =\sum_{v} \varepsilon\left(\mu^{-1} v\right)\left(\sum_{\mu}(v(\xi), \lambda)^{d} \Phi_{\mu}\right) \\
& =\left(\sum_{\mu} \varepsilon(\mu) \Phi_{\mu}\right)\left(\sum_{v} \varepsilon(v)(v(\xi), \lambda)^{d}\right) \\
& =C_{N} \delta(\lambda) \delta(\xi) \psi .
\end{aligned}
$$

Here we used the identity

$$
\begin{aligned}
\sum_{\sigma} \varepsilon(\sigma)(\sigma(\xi), \lambda)^{d} & =C_{N} \prod_{i<j}\left(\lambda_{i}-\lambda_{j}\right) \prod_{i<j}\left(\xi_{i}-\xi_{j}\right)=C_{N} \delta(\lambda) \delta(\xi) \\
C_{N} & =\frac{d !}{1 ! 2 ! 3 ! \ldots(N-1) !}
\end{aligned}
$$

Theorem 1 is proven.

\section{Generalized KZ Equations and Calogero-Sutherland Problems, Related to Root Systems}

The generalizations of Calogero-Sutherland problem we are going to discuss were considered firstly by Olshanetsky and Perelomov [3]. Corresponding versions of the $\mathrm{KZ}$ equation were introduced by Cherednik (see [15-19]). 
Recall (see [20]) that a root system is a finite subset $\Sigma$ of the Euclidean space $E$ with inner product (, ), such that

1) $\Sigma$ does not contain 0 and spans $E$;

2) $s_{\alpha}(\beta) \in \Sigma$ for all $\alpha, \beta \in \Sigma$, where $s_{\alpha}$ is the reflection

$$
s_{\alpha}(x)=x-\left(x, \alpha^{\vee}\right) \alpha, \quad \alpha^{\vee}=2 \alpha /(\alpha, \alpha) ;
$$

3) $\left(\alpha^{\vee}, \beta\right) \in \mathbb{Z}$ for all $\alpha, \beta \in \Sigma$.

The Weyl group $W$ is defined as the subgroup of $G L(E)$ generated by the reflections $s_{\alpha}, \alpha \in \Sigma$. From the second property of root systems it follows that $W$ is a finite group.

The following generalizations of the Sutherland operators related to $\Sigma$ were introduced in [3]:

$$
L_{\Sigma, k}^{S}=-\Delta+\sum_{\alpha \in \Sigma_{+}} k_{\alpha}\left(k_{\alpha}-1\right) \omega^{2}(\alpha, \alpha) \sinh ^{-2} \omega(\alpha, z)
$$

Here $\Delta$ means the laplacian on $E, k_{\alpha}=k(\alpha)$, where $k$ is a $W$-invariant function on $\Sigma$, $\Sigma_{+}$is the set of positive roots of $\Sigma$ for some choice of Weyl chamber [20], $\omega$ is a scalar parameter. In the limit $\omega \rightarrow 0$ one has the generalized Calogero operator

$$
L_{\Sigma, k}^{C}=-\Delta+\sum_{\alpha \in \Sigma_{+}} k_{\alpha}\left(k_{\alpha}-1\right)(\alpha, \alpha)(\alpha, z)^{-2} \text {. }
$$

In this rational limit one can use also any Coxeter group, i.e. one can omit the third property in the definition of root systems (see $[4,10])$.

The operators (2.1) generate a commutative ring of differential operators on $E$ :

$$
\mathscr{R}_{\Sigma, k}^{S}=\left\{\mathscr{A}:\left[\mathscr{A}, L_{\Sigma, k}^{S}\right]=0, w^{*}(\mathscr{A})=\mathscr{A} \text { for all } w \in W\right\} .
$$

These rings are isomorphic to the rings $\mathbb{C}[\lambda]^{W}$ of $W$-invariant polynomials on $E$ (Harish-Chandra isomorphism, see $[5,11]$ ):

$$
\gamma: \mathscr{R}_{\Sigma, k}^{S} \rightarrow \mathbb{C}[\lambda]^{W}
$$

The existence of shift operators for such generalizations of Sutherland operator $L_{\Sigma, k}^{S}$ was proven by Opdam [6]. More precisely, for any $W$-invariant subset $\mathscr{E} \subset \Sigma$ there exists a differential operator $\mathscr{D}_{\mathscr{E}}(k)$, which has the highest order term

$$
\prod_{\alpha \in \mathscr{E}_{+}}(\alpha, \partial), \quad \mathscr{E}_{+}=\mathscr{E} \cap \Sigma_{+}
$$

and satisfies the relation

$$
L_{\Sigma, k+1_{\mathscr{\delta}}}^{S} \mathscr{D}_{\mathscr{E}}(k)=\mathscr{D}_{\mathscr{E}}(k) L_{\Sigma, k}^{S} .
$$

Here $1_{\mathscr{E}}$ denotes the characteristic function of $\mathscr{E}: 1_{\mathscr{E}}(\alpha)=1$ if $\alpha \in \mathscr{E}$ and 0 otherwise (see $[6,7]$ ).

Now let us consider the following version of the $\mathrm{KZ}$ equation $(1.1)$ (see $[15,19])$ :

$$
\partial_{\xi} \Phi=\left(\sum_{\alpha \in \Sigma_{+}} \omega k_{\alpha}(\alpha, \xi)\left(\operatorname{coth} \omega(\alpha, z) \sigma_{\alpha}+\sigma_{\alpha} \varepsilon_{\alpha}\right)+e_{\xi}(\lambda)\right) \Phi(z) .
$$


Here $\Phi$ is a $\mathbb{C}[W]$-valued function, where $\mathbb{C}[W]$ is the Weyl group algebra, $\varepsilon_{\alpha}, \sigma_{\alpha}$, $e_{\xi}(\lambda)$ are the operators on $\mathbb{C}[W]$, defined by

$$
\begin{aligned}
\sigma_{\alpha}[w] & =\left[s_{\alpha} w\right], \\
\varepsilon_{\alpha}[w] & =\left\{\begin{aligned}
{[w] } & \text { if } w^{-1} \alpha \in \Sigma_{+}, \\
-[w] & \text { if } w^{-1} \alpha \in \Sigma_{-},
\end{aligned}\right. \\
e_{\xi}(\lambda)[w] & =(w \lambda, \xi)[w], \quad \xi, \lambda \in E,
\end{aligned}
$$

$k_{\alpha}=k(\alpha), k$ is any $W$-invariant function on $\Sigma$. We used here the notations of Matsuo's paper [15].

Let us consider a character $\varepsilon: W \rightarrow\{ \pm 1\}$. It is determined by the $W$-invariant subset $\mathscr{E} \subset \Sigma$, such that $\varepsilon\left(s_{\alpha}\right)=-1$ if $\alpha \in \mathscr{E}$ and $\varepsilon\left(s_{\alpha}\right)=+1$ otherwise.

Proposition (Matsuo [15], Cherednik [19]). If $\Phi=\sum_{w \in W} \Phi_{w}[w]$ is a solution of the generalized $\mathrm{KZ}$ equation (2.7), then the functions

and

$$
\varphi=\mathrm{m}(\Phi):=\sum_{w \in W} \Phi_{w}
$$

$$
\psi=\operatorname{ch}_{\mathscr{E}}(\Phi):=\sum_{w \in W} \varepsilon(w) \Phi_{w}
$$

are eigenfunctions of the corresponding generalized Sutherland operators:

$$
\begin{aligned}
& \left(-\Delta+\sum_{\alpha \in \Sigma_{+}} \frac{k_{\alpha}\left(k_{\alpha}-1\right)(\alpha, \alpha) \omega^{2}}{\sinh ^{2} \omega(\alpha, z)}\right) \varphi=E \varphi, \\
& \left(-\Delta+\sum_{\alpha \in \Sigma_{+}} \frac{k_{\alpha}^{\varepsilon}\left(k_{\alpha}^{\varepsilon}-1\right)(\alpha, \alpha) \omega^{2}}{\sinh ^{2} \omega(\alpha, z)}\right) \psi=E \psi,
\end{aligned}
$$

where $k_{\alpha}^{\varepsilon}=\left(k+1_{\mathscr{E}}\right)(\alpha)=k_{\alpha}+1$ if $\alpha \in \mathscr{E}$ and $k_{\alpha}$ otherwise, $E=-(\lambda, \lambda)$. Moreover, $\varphi$ and $\psi$ are common eigenfunctions of the corresponding commutative rings $\mathscr{R}_{\Sigma, k}^{S}$ and $\mathscr{R}_{\Sigma, k}^{S} \varepsilon$.

Let $S_{\Sigma}(k)$ and $S_{\Sigma}\left(k+1_{\mathscr{E}}\right)$ be the eigenspaces of these commutative rings:

$$
\begin{aligned}
S_{\Sigma}(k) & =\left\{\varphi: \mathscr{A} \varphi=\gamma(\mathscr{A}) \varphi, \mathscr{A} \in \mathscr{R}_{\Sigma, k}^{S}\right\}, \\
S_{\Sigma}\left(k+1_{\mathscr{E}}\right) & =\left\{\psi: \mathscr{A} \psi=\gamma(\mathscr{A}) \psi, \mathscr{A} \in \mathscr{R}_{\Sigma, k+1_{\mathscr{E}}}^{S}\right\},
\end{aligned}
$$

and $\mathrm{KZ}_{\Sigma}(k)$ be the space of all solutions of the generalized $\mathrm{KZ}$ equation (2.7).

Theorem 2. The following diagram is commutative

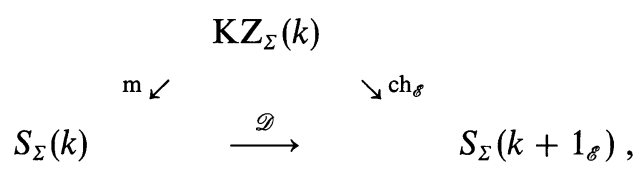

where $\mathscr{D}=\mathscr{D}_{\mathscr{E}}(k) / \prod_{\alpha \in \mathscr{E}_{+}}(\alpha, \lambda+\omega \rho), \rho=\frac{1}{2} \sum_{\alpha \in \Sigma_{+}} k_{\alpha} \alpha$, and $\mathscr{D}_{\mathscr{E}}(k)$ is the shift operator for the generalized Sutherland system.

The proof of this theorem is rather straightforward and is based on the formulas of the papers $[11,15,19]$. We omit it here because it is similar to that of Theorem 1. 
Remark. In the rational case $(\omega=0)$ the corresponding generalization of Theorem 2 is true for any (not necessary crystallographic) Coxeter group.

As a corollary we have the following interpretation of the shift operator for the usual Sutherland operator.

Let us consider the following version of $\mathrm{KZ}$ equation (1.1),

$$
\partial_{i} \Phi=\left[k \omega\left(\sum_{j \neq i} \operatorname{coth} \omega\left(z_{i}-z_{j}\right) P^{(i j)}+T^{(i j)}\right)+\lambda^{(i)}\right] \Phi,
$$

where $\Phi$ takes values in $V^{\otimes N}, \lambda^{(i)}$ is the operator in $V^{\otimes N}$ acting on $i^{\text {th }}$ factor as the diagonal matrix $\lambda=\operatorname{diag}\left(\lambda_{1}, \ldots, \lambda_{n}\right)$ and identically on all other factors, $P$ is the permutation: $P(a \otimes b)=b \otimes a, T$ is the following operator on $V \otimes V$ :

$$
T=\sum_{i>j}\left(E_{i j} \otimes E_{j i}-E_{j i} \otimes E_{i j}\right),
$$

where $E_{i j}$ denotes the matrix with the only non-zero entry 1 at $i j^{\text {th }}$ place, and $P^{(i j)}$ and $T^{(i j)}$ are the corresponding operators in $V^{\otimes N}$ acting only on the $i^{\text {th }}$ and $j^{\text {th }}$ factors. In the limit $\omega \rightarrow 0$ one has the KZ equation (1.1).

Suppose that $\operatorname{dim} V=N$ and consider the solution $\Phi$ of the form

$$
\Phi=\sum_{\sigma \in S_{N}} \Phi_{\sigma} e_{\sigma}, \quad e_{\sigma}=e_{\sigma(1)} \otimes \cdots \otimes e_{\sigma(N)} .
$$

It is easy to check that the corresponding system (2.10), (2.12) can be rewritten in the form (2.7) for $\Sigma$ being the root system of type $A_{N-1}, W=S_{N}, k_{\alpha} \equiv k$, and $e_{\sigma}$ is identified with $\left[\sigma^{-1}\right]$. In this case we have only one nontrivial character $\varepsilon(w)=\operatorname{sign}(w)$, which corresponds to $\mathscr{E}=\Sigma$.

Corollary. If $\Phi$ is a solution of the trigonometric $\mathrm{KZ}$ equation (2.10) of the form (2.12) then the functions

$$
\varphi=\mathrm{m}(\Phi):=\Sigma_{\sigma} \Phi_{\sigma},
$$

and

$$
\psi=\operatorname{ch}(\Phi):=\sum_{\sigma}(\operatorname{sign} \sigma) \Phi_{\sigma}
$$

satisfy the Sutherland equations

$$
\begin{gathered}
\left(-\Delta+k(k-1) \sum_{i \neq j} \omega^{2} \sinh ^{-2} \omega\left(z_{i}-z_{j}\right)\right) \varphi=E \varphi, \\
\left(-\Delta+k(k+1) \sum_{i \neq j} \omega^{2} \sinh ^{-2} \omega\left(z_{i}-z_{j}\right)\right) \psi=E \psi, \quad E=-\lambda^{2},
\end{gathered}
$$

and are related by the formula

$$
\psi=\delta_{\omega k}^{-1}(\lambda) \mathscr{D}_{k}^{S} \varphi,
$$

where $\mathscr{D}_{k}^{S}$ is the shift operator and

$$
\delta_{\omega k}(\lambda)=\prod_{i<j}\left(\lambda_{i}-\lambda_{j}-\omega k(i-j)\right) .
$$

Replacing $\omega \rightarrow \sqrt{-1} \omega$ one has the sin-version of this result.

Notice that $\delta_{\omega k}(\lambda) \neq 0$ is a necessary and sufficient condition for the Matsuo mapping to be an isomorphism (see $[15,19]$ ). 


\section{Inversion of the Matsuo Mapping and the Shift Operators for the KZ Equation}

In this paragraph for simplicity we restrict our consideration to the rational case only, but it can be done in the trigonometric case as well.

So we consider the solutions of $\mathrm{KZ}$ equation (1.1),

$$
\partial_{i} \Phi=\left(k \sum_{j \neq i} \frac{P^{(i j)}}{z_{i}-z_{j}}+\lambda^{(i)}\right) \Phi
$$

of the form $\Phi=\sum_{\sigma \in S_{N}} \Phi_{\sigma} e_{\sigma}$, and Calogero quantum systems $L_{k}^{C} \varphi=E \varphi$,

$$
L_{k}^{C}=\left(-\Delta+k(k-1) \sum_{i \neq j} \frac{1}{\left(z_{i}-z_{j}\right)^{2}}\right) \text {. }
$$

From the results of Sect. 1 we have the following commutative diagram:

Diagram 2

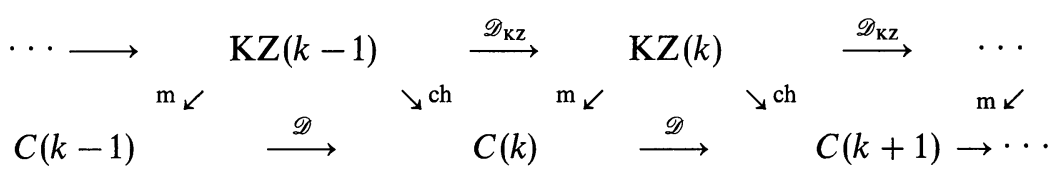

We know from $[15,19]$ that $m$ and ch are isomorphisms iff $\delta(\lambda)=\prod_{i<j}\left(\lambda_{i}-\lambda_{j}\right) \neq 0$. This means that for the generic $\lambda$ one has the shift operator $D_{\mathrm{KZ}}: \mathrm{KZ}(k) \rightarrow \mathrm{KZ}(k+1)$ (see diagram 2 ), which is defined as

$$
\mathscr{D}_{\mathrm{KZ}}=\mathrm{m}^{-1} \circ \mathrm{ch} \text {. }
$$

So to give the explicit expression for this operator we need the inversion of Matsuo mapping $\mathrm{m}$. Now we want to give some formula for this inversion.

Let $P(\xi, \lambda, \eta)$ and $P_{\sigma}(\xi, \lambda, \eta)$ be the following polynomials on $\xi, \lambda, \eta \in \mathbb{R}^{N}$ :

$$
\begin{gathered}
P(\xi, \lambda, \eta)=\prod_{w \in W}\left(\xi-w^{-1}(\lambda), \eta\right), \\
P_{\sigma}(\xi, \lambda, \eta)=\prod_{w \neq \sigma}\left(\xi-w^{-1}(\lambda), \eta\right)=\frac{P(\xi, \lambda, \eta)}{\left(\xi-\sigma^{-1}(\lambda), \eta\right)} .
\end{gathered}
$$

Here $W=S_{N}, \sigma \in W$ is any permutation, ( , ) means the standard scalar product on $\mathbb{R}^{N}$. One can rewrite also

$$
\begin{aligned}
P(\xi, \lambda, \eta) & =\prod_{w}\left(x-\left(w^{-1}(\lambda), \eta\right)\right) . \\
P_{\sigma}(\xi, \lambda, \eta) & \left.=\prod_{w \neq \sigma}\left(x-\left(w^{-1}(\lambda), \eta\right)\right), \quad x=(\xi, \eta)\right) .
\end{aligned}
$$

This allows us to define the operator

$$
P_{\sigma}(\nabla, \lambda, \eta)=\prod_{w \neq \sigma}\left(\nabla_{\eta}-\left(w^{-1}(\lambda), \eta\right)\right),
$$

where $\nabla_{\eta}$ is the Dunkl differential-difference operator (1.16):

$$
\nabla_{\eta}=\partial_{\eta}-k \sum_{j \neq i} \frac{\eta_{i}-\eta_{j}}{z_{i}-z_{j}} \hat{p}_{i j}
$$


Recall that, for such operators $\mathscr{A}$, Res $\mathscr{A}$ denotes the differential part of $\mathscr{A}$, which can be found as restriction of $\mathscr{A}$ to the space of $W$-invariant functions (see Sect. 1 and [10]).

Let $\Phi=\sum_{\sigma} \Phi_{\sigma} e_{\sigma}$ be a solution of $\mathrm{KZ}$ equation (3.1) with regular $\lambda_{:} \lambda_{i} \neq \lambda_{j}$ for all $i \neq j$. We will say that $\eta \in \mathbb{R}^{N}$ is generic if

$$
\left(\eta, w_{1}(\lambda)-w_{2}(\lambda)\right) \neq 0
$$

for all $w_{1}, w_{2} \in W$ such that $w_{1} \neq w_{2}$.

Let

$$
\varphi=m(\Phi)=\sum_{\sigma} \Phi_{\sigma}
$$

Theorem 3. The solution $\Phi=\Sigma \Phi_{\sigma} e_{\sigma}$ of $\mathrm{KZ}$ equation (3.1) for regular $\lambda$ can be reconstructed from $\varphi=m(\Phi)$ by the following formula:

$$
\Phi_{\sigma}=\operatorname{Res}\left(\frac{P_{\sigma}(\nabla, \lambda, \eta)}{P_{\sigma}\left(\sigma^{-1}(\lambda), \lambda, \eta\right)}\right) \varphi
$$

for any generic $\eta$.

Proof. We will use the formula (1.21) from the first paragraph:

$$
\operatorname{Res}\left(\nabla_{\eta}^{m}\right)(\varphi)=\sum_{\mu \in W}(\mu(\eta), \lambda)^{m} \Phi_{\mu}
$$

It implies that

$$
\operatorname{Res}\left(P_{\sigma}(\nabla, \lambda, \eta)\right)(\varphi)=\sum_{\mu} P_{\sigma}\left(\mu^{-1}(\lambda), \lambda, \eta\right) \Phi_{\mu}
$$

But

$$
P_{\sigma}\left(\mu^{-1}(\lambda), \lambda, \eta\right)=\prod_{\tau \neq \sigma}\left(\mu^{-1}(\lambda)-\tau^{-1}(\lambda), \eta\right)=0 \quad \text { if } \mu \neq \sigma,
$$

and $P_{\sigma}\left(\sigma^{-1}(\lambda), \lambda, \eta\right)=\prod_{\tau \neq \sigma}\left(\sigma^{-1}(\lambda)-\tau^{-1}(\lambda), \eta\right) \neq 0$ for generic $\eta$.

So we have

$$
\operatorname{Res}\left(P_{\sigma}(\nabla, \lambda, \eta)(\varphi)\right)=P_{\sigma}\left(\sigma^{-1}(\lambda), \lambda, \eta\right) \Phi_{\sigma},
$$

which proves the theorem.

Let $\mathscr{A}_{\sigma}$ be the operator,

$$
\mathscr{A}_{\sigma}=\operatorname{Res} \frac{P_{\sigma}(\nabla, \lambda, \eta)}{P_{\sigma}\left(\sigma^{-1}(\lambda), \lambda, \eta\right)} .
$$

Corollary 1. The solution $\Phi=\Sigma \Phi_{\sigma} e_{\sigma}$ of $\mathrm{KZ}$ equation (3.1) for regular $\lambda$ can be reconstructed from $\psi=\operatorname{ch} \Phi=\Sigma \varepsilon(\sigma) \Phi_{\sigma}$ by the following formula: $\Phi_{\sigma}=\varepsilon(\sigma) \mathscr{A}_{\sigma} \psi$ for any generic $\eta$.

Corollary 2. The operator $\mathscr{D}_{\mathrm{Kz}}=\mathrm{m}^{-1} \circ \mathrm{ch}$,

$$
\mathscr{D}_{\mathrm{KZ}}(\Phi)=\sum_{\sigma}\left(\sum_{\mu} \varepsilon(\mu) \mathscr{A}_{\sigma} \Phi_{\mu}\right) e_{\sigma}
$$

is a shift operator for $\mathrm{KZ}$ equation

$$
\mathscr{D}_{\mathrm{KZ}}: \mathrm{KZ}(k-1) \rightarrow \mathrm{KZ}(k) .
$$




\section{Factorizations of Discriminant and Shift Operators}

In the introduction it was shown that in the simplest case $N=2$ the shift operator $\mathscr{D}_{k}$ can be found by solving an appropriate factorization problem

$$
L=\mathscr{D}_{k}^{*} \mathscr{D}_{k}
$$

(see the formula (0.4)). The following natural question arises ${ }^{3}$ : is it possible to determine the shift operator in such a way for general $N$ ?

The answer is "yes." To do it we should choose some special operator $L_{\Delta}$ from the commutative ring $\mathscr{R}_{k}^{C}$, which we will call discriminant.

Recall that the ring $\mathscr{R}_{k}^{C}$ of quantum integrals of the Calogero system is isomorphic to the ring of symmetric polynomials

$$
\gamma: \mathscr{R}_{k}^{C} \rightarrow \mathbb{C}[\lambda]^{S_{N}}
$$

The operator $L$ we need corresponds to the symmetric function $\Delta(\lambda)$,

$$
\Delta(\lambda)=\prod_{i \neq j}\left(\lambda_{i}-\lambda_{j}\right)=(-1)^{d} \prod_{i<j}\left(\lambda_{i}-\lambda_{j}\right)^{2}= \pm \delta^{2}(\lambda),
$$

where, as above, $d=N(N-1) / 2$. Let us denote $\gamma^{-1}(\Delta)$ as $L_{\Delta}(k)$.

Theorem 4. The shift operator $\mathscr{D}_{k}$ for the Calogero system with $N$ particles satisfies the following relations with discriminant operator $L_{\Delta}$ :

$$
\begin{aligned}
& \mathscr{D}_{k}^{*} \mathscr{D}_{k}=L_{\Delta}(k), \\
& \mathscr{D}_{k} \mathscr{D}_{k}^{*}=L_{\Delta}(k+1) .
\end{aligned}
$$

$\mathscr{D}_{k}^{*}$ is the operator formally adjoint to $\mathscr{D}_{k}$.

The proof follows from the results of the first paragraph. Let us notice that the diagram 1 can be extended in the following way:

Diagram 3

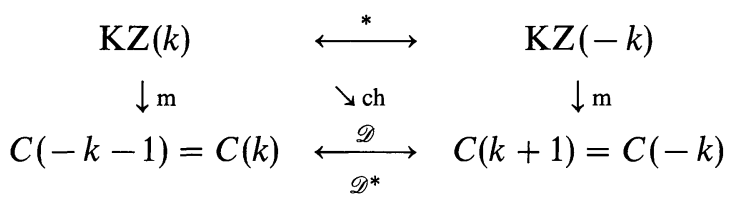

Here the duality $*$ is defined as the involution

$$
* \Phi:=\sum_{\sigma} \varepsilon(\sigma) \Phi_{\sigma} e_{\sigma},
$$

where $\Phi=\Sigma \Phi_{\sigma} e_{\sigma}, \varepsilon(\sigma)$ is the sign of $\sigma \in S_{N}$. It is easy to check that

$$
*: \mathrm{KZ}(k) \rightarrow \mathrm{KZ}(-k) \text {. }
$$

The second remark is that $C(k+1)=C(-k), C(k)=C(-k-1)$ and

$$
\mathscr{D}_{k}^{*}=(-1)^{d} \mathscr{D}_{-k} \text {. }
$$

\footnotetext{
${ }^{3}$ We are grateful to Prof. J. Moser for this question and the stimulating discussions
} 
So we have from diagram 4 , that $\mathscr{D}^{*} \mathscr{D}$ is the identity operator on $C(k)$. By Theorem 1,

$$
\mathscr{D}=\delta^{-1}(\lambda) \mathscr{D}_{k},
$$

which gives relation (4.3). Relation (4.4) follows from the same arguments.

In the trigonometric case (for the Sutherland system) we should take

$$
\Delta_{\omega k}=\delta_{\omega k}^{+} \delta_{\omega k}^{-},
$$

where

$$
\delta_{\omega k}^{ \pm}=\prod_{i<j}\left(\lambda_{i}-\lambda_{j} \pm k \omega(i-j)\right)
$$

and the corresponding operator $L_{\Delta}^{\omega} \in \mathscr{R}_{k}^{S}$. For the generalizations related to the root system $\Sigma$ and the character $\varepsilon$ (see $\S 2$ )

$$
\begin{aligned}
\Delta_{\varepsilon, k \omega} & =\delta_{\Sigma, k \omega}^{+} \delta_{\bar{\varepsilon}, k \omega}^{-}, \\
\delta_{\varepsilon, k \omega}^{ \pm} & =\prod_{\alpha \in \mathscr{E}_{+}}(\lambda \pm \omega \rho, \alpha) .
\end{aligned}
$$

So even in the general case the shift operator $\mathscr{D}_{k}$ for the Calogero-Sutherland problem can be found as the solution of the factorization problem (4.3). It would be interesting to investigate this possibility in more detail.

Acknowledgements. One of the authors (A.V) would like to express his gratitude to the Forschungsinstitut für Mathematik, ETH Zürich, for the hospitality during the winter semester 1992/93.

\section{References}

1. Calogero, F.: Solution of the one-dimensional $N$-body problem with quadratic and/or inversely quadratic pair potentials. J Math. Phys 12, 419-439 (1971)

2. Sutherland, B.: Exact results for a quantum many-body problem in one dimension. Phys. Rev. A4, 2019-2021 (1971); Phys. Rev. A5, 1372-1376 (1972)

3. Olshanetsky, M.A., Perelomov, A.M : Quantum completely integrable systems connected with semi-simple Lie algebras. Lett. Math. Phys. 2, 7-13 (1977)

4. Olshanetsky, M.A., Perelomov, A.M.: Quantum integrable systems related to Lie algebras. Phys. Rep. 94, N6, 313-404 (1983)

5. Heckman, G., Opdam, E.M.: Root systems and hypergeometric functions I, II. Comp. Math. 64, 329-352, 353-373 (1987)

6. Opdam, E.M.: Root systems and hypergeometric functions III, IV. Comp. Math. 67, 21-49, 191-209 (1988)

7. Opdam, E.M.: Some applications of hypergeometric shift operators. Invent. Math. 98, 1-18 (1989)

8. Chalykh, O.A., Veselov, A.P.: Commutative rings of partial differential operators and Lie algebras. Preprint of FIM (ETH, Zürich), 1988; Commun. Math. Phys. 126, 597-611 (1990)

9. Chalykh, O.A., Veselov, A.P.: Integrability in the theory of Schrödinger operator and harmonic analysis. Preprint of FIM (ETH, Zürich), 1992. To appear in Commun. Math. Phys.

10. Heckman, G.: A remark on the Dunkl differential-difference operators. Progress in Math. 101, 181-191 (1991)

11. Heckman, G.: An elementary approach to the hypergeometric shift operators of Opdam. Invent. Math. 103, 341-350 (1991)

12. Dunkl, C.F.: Differential-difference operators associated to reflection groups. Trans. AMS 311, 167-183 (1989) 
13 Knizhnik, V.G., Zamolodchikov, A.B.: Current algebra and Wess-Zumino models in two dimensions. Nucl. Phys. B247, 83-103 (1984)

14. Moser, J.: Three integrable Hamiltonian systems, connected with isospectral deformations. Adv. Math. 16, 1-23 (1978)

15. Matsuo, A.: Integrable connections related to zonal spherical function. Inv. Math. 110, 95-121 (1992)

16. Cherednik, I.V.: Generalized braid groups and local 2-matrix systems. Doklady AN SSR, 307:1, 27-34 (1989)

17. Cherednik, I.V.: Monodromy representations for generalized $\mathrm{KZ}$ equations and Hecke algebras. Preprint ITP-89-74E (Kiev, 1989)

18. Cherednik, I.V.: A unification of $\mathrm{KZ}$ and Dunkl operators via affine Hecke algebras. Invent. Math. 106, 411-431 (1991)

19. Cherednik, I.V.: Integration of quantum many-body problems by affine $\mathrm{KZ}$ equations. Preprint of RIMS, Kyoto, 1991

20. Bourbaki, N.: Groupes et algèbres de Lie. Chapitres 4-6. Paris: Hermann 1969

Communicated by A. Jaffe 
